\title{
SHARP DISTORTION GROWTH FOR BILIPSCHITZ EXTENSION OF PLANAR MAPS
}

\author{
LEONID V. KOVALEV
}

Abstract. This note addresses the quantitative aspect of the bilipschitz extension problem. The main result states that any bilipschitz embedding of $\mathbb{R}$ into $\mathbb{R}^{2}$ can be extended to a bilipschitz self-map of $\mathbb{R}^{2}$ with a linear bound on the distortion.

\section{INTRODUCTION}

Let $X$ and $Y$ be metric spaces, with distance denoted by $|\cdot|$. A map $f: X \rightarrow Y$ is an $(L, \ell)$-bilipschitz embedding if

$$
\ell|a-b| \leq|f(a)-f(b)| \leq L|a-b| \quad \text { for all } \quad a, b \in X .
$$

If $f$ is, in addition, surjective, it is a bilipschitz isomorphism between $X$ and $Y$ (an automorphism if $X=Y$ ). In this paper $X$ and $Y$ will be subsets of the plane $\mathbb{R}^{2} \simeq \mathbb{C}$. The following theorem was proved by Tukia in $[22,23$; see also [11, 14].

Theorem 1.1. Any $(L, \ell)$-bilipschitz embedding $f: \mathbb{R} \rightarrow \mathbb{C}$ can be extended to an $\left(L^{\prime}, \ell^{\prime}\right)$-bilipschitz automorphism $F: \mathbb{C} \rightarrow \mathbb{C}$, where $L^{\prime}, \ell^{\prime}$ depend only on $L$ and $\ell$.

Conjugating $f$ by a Möbuis map [23, p. 93], one obtains a version of Theorem 1.1 for bilipschitz embeddings $f: \mathbb{T} \rightarrow \mathbb{C}$, where $\mathbb{T}$ is the unit circle $\{|z|=1\}$. The aforementioned papers do not provide explicit estimates for $L^{\prime}$ and $\ell^{\prime}$, although such estimates could in principle be obtained by following the approach of [11, 23.

For embeddings of $\mathbb{T}$, Daneri and Pratelli [7] recently achieved $L^{\prime}=C L^{4}$ and $\ell^{\prime}=\ell^{4} / C$ with a universal constant $C$. They asked whether $L^{\prime}$ and $\ell^{\prime}$ can have linear growth, that is $L^{\prime}=C L$ and $\ell^{\prime}=\ell / C$ with universal $C$. We prove this for embeddings of $\mathbb{R}$.

Theorem 1.2. Any $(L, \ell)$-bilipschitz embedding $f: \mathbb{R} \rightarrow \mathbb{C}$ can be extended to an $\left(L^{\prime}, \ell^{\prime}\right)$-bilipschitz automorphism $F: \mathbb{C} \rightarrow \mathbb{C}$, where $L^{\prime}=2000 L$ and $\ell^{\prime}=\ell / 120$.

Theorem 1.2 appears to be the first bilipschitz extension result with linear growth of distortion. This contrasts with the state of our knowledge of Lipschitz extensions, for which the linear bound $L^{\prime} \leq C L$ is usually available [6]. In the Euclidean setting one even has a Lipschitz extension with $L^{\prime}=L$, by Kirszbraun's theorem [13.

In the case of small distortion, $L / \ell \approx 1$, Tukia and Väisälä [25, Theorem 5.3] used a triangulation method to construct an extension such that $L^{\prime} / \ell^{\prime} \rightarrow 1$ as $L / \ell \rightarrow 1$; moreover, their construction works in all dimensions. In contrast to linear growth in Theorem 1.2, the decay of $L^{\prime} / \ell^{\prime}-1$ cannot be linear. The best possible estimate

Received by the editors March 15, 2012.

2010 Mathematics Subject Classification. Primary 26B35; Secondary 57N35, 51F99, 54C25.

Key words and phrases. Bilipschitz extension, conformal map.

Supported by the NSF grant DMS-0968756. 
is of order $(L / \ell-1)^{1 / 2}$ [25, Example 5.12]. The sets that allow for extensions with linear decay of the distortion were recently studied in [2, 3, 21]. The extension used in the proof of Theorem 1.2 does not have the property $L^{\prime} / \ell^{\prime} \rightarrow 1$ as $L / \ell \rightarrow 1$. For example, the identity map is extended to $F(x+i y)=x+i y / 2$. It is conceivable that one could achieve $L^{\prime} / \ell^{\prime} \rightarrow 1$ with a modified construction and more elaborate estimates, but this would come at the cost of a substantially longer proof (cf. [5]).

In order to achieve the linear growth bound, the proof of Theorem 1.2 must be structured so that the bilipschitz property of $f$ is used only once. Thus the proof is necessarily different from the earlier ones. For instance, it does not rely on the fact that conformal maps between quasidisks are quasisymmetric. Instead, it is based on the Beurling-Nevanlinna theorem for harmonic measure [10, 19]. The other key tools are the Riemann mapping theorem and the Beurling-Ahlfors extension, as in 23 .

Conjugating $f$ and $F$ by a global bilipschitz map yields a corollary for unbounded chordarc curves, i.e., images of $\mathbb{R}$ under bilipschitz embeddings into $\mathbb{C}$.

Corollary 1.3. For any unbounded chordarc curve $\Gamma \subset \mathbb{C}$ there exists a constant $C$ such that any $(L, \ell)$-bilipschitz embedding $f: \Gamma \rightarrow \mathbb{C}$ can be extended to a $(C L, \ell / C)$ bilipschitz automorphism $F: \mathbb{C} \rightarrow \mathbb{C}$.

Remark 1.4. In the literature it is common to use $1 / L$ in place of $\ell$ in the formula (1.1) and call $f$ an $L$-bilipschitz map. This introduces a symmetry between expansion and contraction, between $f$ and $f^{-1}$. However, such symmetry is lacking in the setting of Theorem 1.2, where the domain of $f$ is a line while the range can be any chordarc curve. Thus, one cannot expect the expansion and contraction constants to deteriorate by the same factor in the process of extension. Indeed, in Theorem 1.2 we have two different factors, 2000 and 120, attached to the expansion and compression constants. On the other hand, these factors are probably far from optimal (cf. Example 5.2), and so their lack of equality does not mean much.

Due to topological obstructions, Theorem 1.1 does not hold in higher dimensions [22]. However, there are several bilipschitz extension results that avoid or overcome such obstructions [4, 8, 12, 15, 16, 17, 20, 24, 26, 27] and it would be interesting to know which of them admit linear control of the distortion. The original question of Daneri and Pratelli, for embeddings of $\mathbb{T}$ into $\mathbb{C}$, also remains open.

The paper is structured as follows. Section 2 collects prerequisite results. In $\$ 3$ we estimate the derivative of a conformal map of halfplane. Bilipschitz condition makes its first appearance in 4 where Theorem 1.2 is proved. In $\$ 5$ we show that some increase of the distortion is inevitable; i.e., one cannot have $\left(L^{\prime}, \ell^{\prime}\right)=(L, \ell)$.

\section{Preliminaries}

Throughout the paper $\mathbb{H}$ denotes the open upper halfplane. The open disk of radius $r$ centered at $a$ is denoted by $B(a, r)$ and its closure by $\bar{B}(a, r)$. We write $\omega(\zeta, E, \Omega)$ for the harmonic measure of a set $E \subset \partial \Omega$ with respect to a point $\zeta$ in a domain $\Omega$.

The following proposition is [19, Corollary 4.5.9]; it can be seen as a corollary of the Beurling-Nevanlinna projection theorem. 
Proposition 2.1. Let $\Omega \subset \mathbb{C} \backslash\{0\}$ be a simply connected domain. Pick a point $\zeta \in \Omega$ and let $\rho>0$.

(a) If $|\zeta|<\rho$, then

$$
\omega(\zeta, \partial \Omega \cap B(0, \rho), \Omega) \geq \frac{2}{\pi} \sin ^{-1}\left(\frac{\rho-|\zeta|}{\rho+|\zeta|}\right) .
$$

(b) If $|\zeta|>\rho$, then

$$
\omega(\zeta, \partial \Omega \cap \bar{B}(0, \rho), \Omega) \leq \frac{2}{\pi} \cos ^{-1}\left(\frac{|\zeta|-\rho}{|\zeta|+\rho}\right) .
$$

In 19 the inequality (2.2) is stated for the open disk $B(0, \rho)$. The version given here follows by replacing $\rho$ with $\rho+\epsilon$ and letting $\epsilon \rightarrow 0$.

The following two-sided derivative bound is a straightforward consequence of the Schwarz lemma and the Koebe $1 / 4$-theorem.

Proposition 2.2. Let $\Phi: \mathbb{H} \rightarrow \Omega \subset \mathbb{C}$ be a conformal map. For any $z \in \mathbb{H}$,

$$
\frac{1}{2} \operatorname{Im} z\left|\Phi^{\prime}(z)\right| \leq \operatorname{dist}(\Phi(z), \partial \Omega) \leq 2 \operatorname{Im} z\left|\Phi^{\prime}(z)\right| .
$$

\section{Distortion of a CONFORMal map}

Lemma 3.1. Suppose that $\Phi: \mathbb{H} \rightarrow \Omega$ is a conformal map that extends continuously to the boundary $\partial \mathbb{H}=\mathbb{R}$. Let $\phi: \mathbb{R} \rightarrow \partial \Omega$ denote the induced boundary map. Fix $x+i y \in \mathbb{H}$ and consider four subsets of $\partial \Omega$ defined as

$$
\begin{aligned}
\Gamma_{1}=\phi((-\infty, x-y]), & \Gamma_{2}=\phi([x-y, x-y / 2]), \\
\Gamma_{3}=\phi([x+y / 2, x+y]), & \Gamma_{4}=\phi([x+y,+\infty)) .
\end{aligned}
$$

Then

$$
\frac{1}{120} \operatorname{dist}\left(\Gamma_{1}, \Gamma_{4}\right) \leq y\left|\Phi^{\prime}(x+i y)\right| \leq 500 \min \left(\operatorname{diam} \Gamma_{2}, \operatorname{diam} \Gamma_{3}\right) .
$$

Proof. Denote $\zeta=\Phi(x+i y)$. In order to prove the first inequality in (3.2) we first show that

$$
\operatorname{dist}\left(\zeta, \Gamma_{j}\right) \leq 30 \operatorname{dist}(\zeta, \partial \Omega), \quad j=1,4 .
$$

By symmetry it suffices to consider $j=1$. Recall that the harmonic measure $\omega$ in the halfplane coincides with the normalized angular measure [10, I.1]. This and the conformal invariance of the harmonic measure imply

$$
\omega\left(\zeta, \Gamma_{1}, \Omega\right)=\omega(x+i y,(-\infty, x-y], \mathbb{H})=\frac{1}{\pi} \frac{\pi}{4}=\frac{1}{4} .
$$

By translation we can achieve $0 \in \partial \Omega$ and $|\zeta|=\operatorname{dist}(\zeta, \partial \Omega)$. Let $\rho=\operatorname{dist}\left(\zeta, \Gamma_{j}\right)$. If $\rho=|\zeta|$, then (3.3) holds. Otherwise $\rho>|\zeta|$ and we can apply (2.1) to obtain

$$
\omega(\zeta, \partial \Omega \cap B(0, \rho), \Omega) \geq \frac{2}{\pi} \sin ^{-1}\left(\frac{\rho-|\zeta|}{\rho+|\zeta|}\right) .
$$

On the other hand, $\partial \Omega \cap B(0, \rho)$ is disjoint from $\Gamma_{1}$, which yields

$$
\omega(\zeta, \partial \Omega \cap B(0, \rho), \Omega) \leq 1-\omega\left(\zeta, \Gamma_{1}, \Omega\right)=\frac{3}{4} .
$$


Combining (3.5) and (3.6) we find that

$$
\frac{\rho-|\zeta|}{\rho+|\zeta|} \leq \sin \frac{3 \pi}{8}, \quad \text { hence } \quad \rho \leq 30|\zeta|
$$

which is 3.3 .

From (3.3) and (2.3) we obtain

$$
\operatorname{dist}\left(\Gamma_{1}, \Gamma_{2}\right) \leq 60 \operatorname{dist}(\zeta, \partial \Omega) \leq 120 y\left|\Phi^{\prime}(x+i y)\right|
$$

completing the proof of the first inequality in (3.2).

To establish the second part of (3.2), it suffices to consider $\Gamma_{2}$, as $\Gamma_{3}$ can be treated similarly. We again use the invariance of harmonic measure,

$$
\omega\left(\zeta, \Gamma_{2}, \Omega\right)=\omega(x+i y,[x-y, x-y / 2], \mathbb{H})=\frac{1}{\pi}\left(\frac{\pi}{4}-\frac{\pi}{6}\right)=\frac{1}{12} .
$$

By translation we can assume $0 \in \Gamma_{2}$. Let $\rho=\operatorname{diam} \Gamma_{2}$. By (3.7),

$$
\omega(\zeta, \partial \Omega \cap \bar{B}(0, \rho), \Omega) \geq \frac{1}{12} .
$$

Comparing the latter bound with (2.2) we conclude that

$$
|\zeta| \leq \rho \frac{1+\cos \frac{\pi}{24}}{1-\cos \frac{\pi}{24}}<250 \rho .
$$

By virtue of (2.3),

$$
y\left|\Phi^{\prime}(x+i y)\right| \leq 2 \operatorname{dist}(\zeta, \partial \Omega) \leq 2|\zeta|<500 \operatorname{diam} \Gamma_{2}
$$

as required.

\section{Proof of Theorem 1.2}

Let $D F$ denote the Jacobian matrix of $F$. Our goal is to construct a homeomorphism $F: \mathbb{C} \rightarrow \mathbb{C}$ that agrees with $f$ on the real axis $\mathbb{R}$, is $C^{1}$-smooth in $\mathbb{C} \backslash \mathbb{R}$, and satisfies the inequalities

$$
\|D F(z)\| \leq 2000 L \quad \text { and } \quad\left\|(D F(z))^{-1}\right\| \leq 120 / \ell
$$

for all $z \in \mathbb{C} \backslash \mathbb{R}$. Indeed, the desired Lipschitz properties of $F$ and $F^{-1}$ follow from (4.1) by integration along line segments.

We focus on extending $f$ to the upper halfplane $\mathbb{H}$, the extension to the lower halfplane being similar. The unbounded simple curve $\Gamma=f(\mathbb{R})$ divides the plane into two unbounded domains. One of them, denoted $\Omega$, corresponds to $\mathbb{H}$ via the boundary orientation induced by $f$. Let $\Phi$ be a conformal map of $\mathbb{H}$ onto $\Omega$ such that $|\Phi(z)| \rightarrow \infty$ as $|z| \rightarrow \infty$. By Carathéodory's theorem, $\Phi$ extends to a homeomorphism between the closures of $\mathbb{H}$ and $\Omega$. We use the lowercase letter $\phi$ for the boundary correspondence $\phi: \mathbb{R} \rightarrow \Gamma$.

Define $\psi: \mathbb{R} \rightarrow \mathbb{R}$ by $\psi=f^{-1} \circ \phi$; this is an increasing homeomorphism of $\mathbb{R}$ onto $\mathbb{R}$. The bilipschitz property of $f$ can now be stated as

$$
\ell|\psi(a)-\psi(b)| \leq|\phi(a)-\phi(b)| \leq L|\psi(a)-\psi(b)|, \quad a, b \in \mathbb{R} .
$$

For future reference we note two consequences of (4.2):

$$
\operatorname{diam} \phi([a, b]) \leq L(\psi(b)-\psi(a)) \quad \text { whenever } a<b,
$$

and

$$
\operatorname{dist}(\phi((-\infty, a]), \phi([b,+\infty))) \geq \ell(\psi(b)-\psi(a)) \quad \text { whenever } a<b
$$


Let $\Psi: \mathbb{H} \rightarrow \mathbb{H}$ denote the Beurling-Ahlfors extension of $\psi$, namely

$$
\Psi(x+i y)=\frac{1}{2} \int_{-1}^{1} \psi(x+t y)(1+i \operatorname{sign} t) d t .
$$

This is a quasiconformal map of $\mathbb{H}$ onto itself [1, 5]. The desired extension $F: \mathbb{H} \rightarrow$ $\Omega$ will be the composition $F=\Phi \circ \Psi^{-1}$. Thus, our goal (4.1) can be restated in terms of $\Psi$ as follows.

$$
\frac{\ell}{120}\|D \Psi(z)\| \leq\left|\Phi^{\prime}(z)\right| \leq \frac{2000 L}{\left\|(D \Psi(z))^{-1}\right\|} .
$$

We fix a point $z=x+i y \in \mathbb{H}$ for the rest of the proof. The partial derivatives of $\Psi$ were computed in [1, p. 43],

$$
D \Psi=\frac{1}{2 y}\left(\begin{array}{ll}
\alpha+\beta & \gamma-\delta \\
\alpha-\beta & \gamma+\delta
\end{array}\right)
$$

where

$$
\begin{array}{ll}
\alpha=\psi(x+y)-\psi(x), & \gamma=\int_{0}^{1}(\psi(x+y)-\psi(x+t y)) d t \\
\beta=\psi(x)-\psi(x-y), & \delta=\int_{-1}^{0}(\psi(x+t y)-\psi(x-y)) d t .
\end{array}
$$

Here $\alpha, \beta, \gamma, \delta>0$ because $\psi$ is increasing. For the same reason, $\alpha>\gamma$ and $\beta>\delta$. It follows that the greatest entry of $D \Psi$ is $\frac{\alpha+\beta}{2 y}$. Hence,

$$
\|D \Psi\| \leq \frac{\alpha+\beta}{y}=\frac{\psi(x+y)-\psi(x-y)}{y} .
$$

We now invoke (4.4) and (3.2) to obtain

$$
\|D \Psi\| \leq \frac{1}{y \ell} \operatorname{dist}\left(\Gamma_{1}, \Gamma_{4}\right) \leq \frac{120}{\ell}\left|\Phi^{\prime}(x+i y)\right|
$$

which is the first inequality in (4.6).

To prove the second inequality in (4.6), we calculate the Jacobian determinant

$$
\operatorname{det} D \Psi=\frac{\alpha \delta+\beta \gamma}{2 y^{2}}
$$

and use (4.10) and (4.9) to estimate

$$
\left\|(D \Psi)^{-1}\right\|=\frac{\|D \Psi\|}{\operatorname{det} D \Psi} \leq \frac{\alpha+\beta}{y} \frac{2 y^{2}}{\alpha \delta+\beta \gamma} \leq \frac{2 y}{\min (\gamma, \delta)} .
$$

A lower estimate on $\gamma$ and $\delta$ is now required. Recall that

$$
\gamma=\int_{0}^{1}(\psi(x+y)-\psi(x+t y)) d t \geq \frac{1}{2}(\psi(x+y)-\psi(x+y / 2)) \geq \frac{1}{2 L} \operatorname{diam} \Gamma_{3}
$$

where the last step is based on (4.3). Similarly, $\delta \geq \frac{1}{2 L} \operatorname{diam} \Gamma_{2}$. The second inequality in (3.2) yields

$$
\min (\gamma, \delta) \geq \frac{1}{1000 L} y\left|\Phi^{\prime}(x+i y)\right|
$$


which we plug into (4.11) to obtain

$$
\left\|(D \Psi)^{-1}\right\| \leq \frac{2000 L}{\left|\Phi^{\prime}\right|}
$$

completing the proof of (4.6) and of the theorem.

Remark 4.1. The construction of $F$ involved a conformal map $\Phi$ which is not determined uniquely. Indeed, the requirement that $F$ maps $\mathbb{H}$ onto $\Omega$ while fixing the boundary point $\infty$ determines $F$ only up to pre-composition with a linear map $\eta=r z+s$, with $r>0$ and $s \in \mathbb{R}$. However, replacing $\Phi$ with $\Phi \circ \eta$ results in replacing $\Psi$ with $\Psi \circ \eta$, and consequently, the composition $F=\Phi \circ \Psi^{-1}$ remains unchanged. Thus, the extended map $F$ depends only on $f$.

In a similar way one observes that the extension process commutes with linear transformations, i.e., the extension of $f \circ \eta$ is $F \circ \eta$, and the extension of $\eta \circ f$ is $\eta \circ F$. However, this commutativity does not hold for general Möbius maps due to the distinguished role of the point $\infty$. It is unclear whether the conformally natural extension constructed by Douady and Earle [9] allows for a linear bound on bilipschitz distortion.

\section{EXAMPles}

By Kirszbraun's theorem, any $L$-Lipschitz map from a subset of $\mathbb{R}^{n}$ into $\mathbb{R}^{n}$ can be extended to an $L$-Lipschitz map of $\mathbb{R}^{n}$. That is, no loss of the Lipschitz constant is incurred in the extension. In this section we show that bilipschitz maps do not always admit such a lossless extension. First consider the embeddings of $\mathbb{T}$.

Example 5.1. Define $f: \mathbb{T} \rightarrow \mathbb{C}$ by

$$
f(x+i y)=|x+1 / 2|+i y .
$$

This map is $(1, \ell)$-bilipschitz for some $\ell \in(0,1)$. However, any bilipschitz extension $F: \mathbb{C} \rightarrow \mathbb{C}$ must have $L^{\prime} \geq \frac{2 \pi}{3 \sqrt{3}}>1$.

Proof. The line segment connecting the points $-1 / 2 \pm i \frac{\sqrt{3}}{2}$ is mapped by $F$ to a curve that connects the points $\pm i \frac{\sqrt{3}}{2}$ and stays within the domain enclosed by $f(\mathbb{T})$. Such a curve must have length at least $2 \pi / 3$.

The idea of Example 5.1 is that folding a curve $\Gamma$ across a line may increase the intrinsic metric in one of the components of $\mathbb{C} \backslash \Gamma$. This trick does not work for $\Gamma=\mathbb{R}$, but we have a different example for this case; cf. [25, Example 5.12].

Example 5.2. Define $f: \mathbb{R} \rightarrow \mathbb{C}$ by

$$
f(x)= \begin{cases}x, & x \geq 0, \\ i x, & x \leq 0 .\end{cases}
$$

This map is $\left(1, \frac{1}{\sqrt{2}}\right)$-bilipschitz, but it does not admit any $(1.1,0.7)$-bilipschitz extension $F: \mathbb{C} \rightarrow \mathbb{C}$.

Proof. Suppose $F$ exists and let $w=F(i)$. From the inequalities,

$$
|w-2| \leq 1.1|i-2|=1.1 \sqrt{5} \quad \text { and } \quad|w+2 i| \leq 1.1|i+2|=1.1 \sqrt{5},
$$

we obtain $\operatorname{Re} w \geq 2-1.1 \sqrt{5}$ and $\operatorname{Im} w \leq 1.1 \sqrt{5}-2$. Therefore, the distance from $w$ to $f(\mathbb{R})$ does not exceed $\sqrt{2}(1.1 \sqrt{5}-2)<0.7$, which is a contradiction because $\operatorname{dist}(i, \mathbb{R})=1$. 
The factor of 1.1 in Example 5.2 is rather modest compared to 2000 in Theorem 1.2. This leaves the possibility that Theorem 1.2 could hold with much better bounds, for example $L^{\prime}=2 L$ and $\ell^{\prime}=\ell / 2$.

\section{ACKNOWLEDGEMENTS}

This paper benefited from discussions at the AIM workshop "Mapping theory in metric spaces" organized by Luca Capogna, Jeremy Tyson and Stefan Wenger. In particular, thanks are due to Mario Bonk, Melkana Brakalova, Colin Carroll, Hrant Hakobyan, Kabe Moen, and Charles Smart.

\section{REFERENCES}

1. L. V. Ahlfors, Lectures on quasiconformal mappings, 2nd ed. Amer. Math. Soc., Providence, RI, 2006. MR2241787 (2009d:30001)

2. P. Alestalo, D. A. Trotsenko and J. Väisälä, The linear extension property of bi-Lipschitz mappings, Sibirsk. Mat. Zh. 44 (2003), no. 6, 1226-1238; translation in Siberian Math. J. 44 (2003), no. 6, 959-968. MR2034930 (2004k:30042)

3. P. Alestalo, D. A. Trotsenko and J. Väisälä, Plane sets allowing bilipschitz extensions, Math. Scand. 105 (2009), no. 1, 134-146. MR2549802 (2010h:26013)

4. J. Azzam and R. Schul, Hard Sard: quantitative implicit function and extension theorems for Lipschitz maps, Geom. Funct. Anal., arXiv:1105.4198.

5. A. Beurling and L. Ahlfors, The boundary correspondence under quasiconformal mappings, Acta Math. 96 (1956), 125-142. MR0086869(19:258c)

6. A. Brudnyi and Yu. Brudnyi, Methods of geometric analysis in extension and trace problems, Birkhäuser, 2011.

7. S. Daneri and A. Pratelli, A planar bi-Lipschitz extension theorem, arXiv:1110.6124.

8. G. David and S. Semmes, Singular integrals and rectifiable sets in $\mathbb{R}^{n}$ : Beyond Lipschitz graphs, Astérisque No. 193 (1991), 152 pp. MR.1113517(92j:42016)

9. A. Douady and C. J. Earle, Conformally natural extension of homeomorphisms of the circle, Acta Math. 157 (1986), no. 1-2, 23-48. MR857678 (87j:30041)

10. J. B. Garnett and D. E. Marshall, Harmonic measure, Cambridge Univ. Press, Cambridge, 2005. MR2150803 (2006g:31002)

11. D. S. Jerison and C. E. Kenig, Hardy spaces, $A_{\infty}$, and singular integrals on chord-arc domains, Math. Scand. 50 (1982), no. 2, 221-247. MR672926 (84k:30037)

12. D. Kalaj, Radial extension of a bi-Lipschitz parametrization of a starlike Jordan curve, arXiv:1011.5204.

13. M. D. Kirszbraun, Über die zusammenziehende und Lipschitzsche Transformationen, Fund. Math. 22 (1934), 77-108.

14. T. G. Latfullin, On geometric conditions for images of lines and circles under planar quasiisometries, in "Proceedings of the XVIII All-Union scientific student conference", Novosibirsk, 1980, 18-22.

15. T. G. Latfullin, Continuation of quasi-isometric mappings, Sibirsk. Mat. Zh. 24 (1983), no. 4, 212-216. MR713600 (84j:30036)

16. A. Lukyanenko, Bi-Lipschitz extension from boundaries of certain hyperbolic spaces, arXiv:1112.2684.

17. P. MacManus, Bi-Lipschitz extensions in the plane, J. Anal. Math. 66 (1995), 85-115. MR.1370347 (97b:30028)

18. Ch. Pommerenke, Boundary behaviour of conformal maps, Springer-Verlag, Berlin, 1992. MR.1217706 (95b:30008)

19. T. Ransford, Potential theory in the complex plane, Cambridge Univ. Press, Cambridge, 1995. MR.1334766 (96e:31001)

20. S. Semmes, Chord-arc surfaces with small constant. II. Good parameterizations, Adv. Math. 88 (1991), no. 2, 170-199. MR1120612 (93d:42019b)

21. D. A. Trotsenko, Extendability of classes of maps and new properties of upper sets, Complex Anal. Oper. Theory 5 (2011), no. 3, 967-984. MR2836337 
22. P. Tukia, The planar Schönflies theorem for Lipschitz maps, Ann. Acad. Sci. Fenn. Ser. A I Math. 5 (1980), no. 1, 49-72. MR595177 (82e:57003)

23. P. Tukia, Extension of quasisymmetric and Lipschitz embeddings of the real line into the plane, Ann. Acad. Sci. Fenn. Ser. A I Math. 86 (1981), 89-94. MR639966 (83d:30022)

24. P. Tukia and J. Väisälä, Lipschitz and quasiconformal approximation and extension, Ann. Acad. Sci. Fenn. Ser. A I Math. 6 (1981), no. 2, 303-342. MR658932 (84a:57016)

25. P. Tukia and J. Väisälä, Extension of embeddings close to isometries or similarities, Ann. Acad. Sci. Fenn. Ser. A I Math. 9 (1984), 153-175. MR752401(85i:30048)

26. P. Tukia and J. Väisälä, Bi-Lipschitz extensions of maps having quasiconformal extensions, Math. Ann. 269 (1984), no. 4, 561-572. MR766014 (86c:30041)

27. J. Väisälä, Bi-Lipschitz and quasisymmetric extension properties, Ann. Acad. Sci. Fenn. Ser. A I Math. 11 (1986), no. 2, 239-274. MR853960(88b:54012)

Department of Mathematics, Syracuse University, Syracuse, New York 13244

E-mail address: lvkovale@syr.edu 